
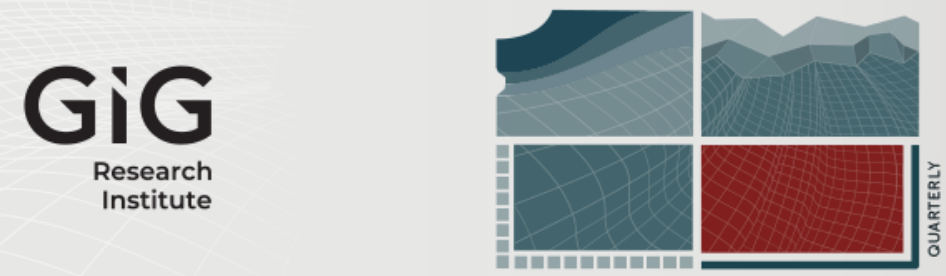

JOURNAL

OF

SUSTAINABLE

MINING

\title{
Evaluating the Performance of Extreme Learning Machine Technique for Ore Grade Estimation
}

Author(s) ORCID Identifier:

Yao Yevenyo Ziggah (iD) 0000-0002-9940-1845

Follow this and additional works at: https://jsm.gig.eu/journal-of-sustainable-mining

Part of the Geological Engineering Commons, and the Mining Engineering Commons

\section{Recommended Citation}

Abuntori, Clara Akalanya; Al-Hassan, Sulemana; Mireku-Gyimah, Daniel; and Ziggah, Yao Yevenyo (2021) "Evaluating the Performance of Extreme Learning Machine Technique for Ore Grade Estimation," Journal of Sustainable Mining: Vol. 20 : Iss. 2 , Article 2.

Available at: https://doi.org/10.46873/2300-3960.1062

This Research Article is brought to you for free and open access by Journal of Sustainable Mining. It has been accepted for inclusion in Journal of Sustainable Mining by an authorized editor of Journal of Sustainable Mining. 


\title{
Evaluating the Performance of Extreme Learning Machine Technique for Ore Grade Estimation
}

\author{
Abstract \\ Due to the complex geology of vein deposits and their erratic grade distributions, there is the tendency of \\ overestimating or underestimating the ore grade. These estimated grade results determine the \\ profitability of mining the ore deposit or otherwise. In this study, five Extreme Learning Machine (ELM) \\ variants based on hard limit, sigmoid, triangular basis, sine and radial basis activation functions were \\ applied to predict ore grade. The motive is that the activation function has been identified to play a key \\ role in achieving optimum ELM performance. Therefore, assessing the extent of influence the activation \\ functions will have on the final outputs from the ELM has some scientific value worth investigating. This \\ study therefore applied ELM as ore grade estimator which is yet to be explored in the literature. The \\ obtained results from the five ELM variants were analysed and compared with the state-of-the-art \\ benchmark methods of Backpropagation Neural Network (BPNN) and Ordinary Kriging (OK). The \\ statistical test results revealed that the ELM with sigmoid activation function (ELM-Sigmoid) was the best \\ among all the other investigated methods (ELM-Hard limit, ELM-Triangular basis, ELM-Sine, ELM-Radial \\ Basis, BPNN and OK). This is because the ELM-sigmoid produced the lowest MAE (0.0175), MSE (0.0005) \\ and RMSE (0.0229) with highest $\mathrm{R}^{2}(91.93 \%)$ and $\mathrm{R}(95.88 \%)$ respectively. It was concluded that ELM- \\ Sigmoid can be used by field practitioners as a reliable alternative ore grade estimation technique. \\ Keywords \\ Extreme learning machine, Artificial intelligence, Artificial neural network, Grade estimation, Kriging \\ Creative Commons License \\ (c) (i) \\ This work is licensed under a Creative Commons Attribution 4.0 License.
}




\title{
Evaluating the performance of Extreme Learning Machine technique for ore grade estimation
}

\author{
Clara Akalanya Abuntori ${ }^{a, *}$, Sulemana Al-Hassan a , \\ Daniel Mireku-Gyimah ${ }^{a}$, Yao Yevenyo Ziggah ${ }^{b}$ \\ ${ }^{\text {a }}$ Department of Mining Engineering, University of Mines and Technology, Tarkwa, Ghana \\ ${ }^{\mathrm{b}}$ Department of Geomatic Engineering, University of Mines and Technology, Tarkwa, Ghana
}

\begin{abstract}
Due to the complex geology of vein deposits and their erratic grade distributions, there is the tendency of overestimating or underestimating the ore grade. These estimated grade results determine the profitability of mining the ore deposit or otherwise. In this study, five Extreme Learning Machine (ELM) variants based on hard limit, sigmoid, triangular basis, sine and radial basis activation functions were applied to predict ore grade. The motive is that the activation function has been identified to play a key role in achieving optimum ELM performance. Therefore, assessing the extent of influence the activation functions will have on the final outputs from the ELM has some scientific value worth investigating. This study therefore applied ELM as ore grade estimator which is yet to be explored in the literature. The obtained results from the five ELM variants were analysed and compared with the state-of-the-art benchmark methods of Backpropagation Neural Network (BPNN) and Ordinary Kriging (OK). The statistical test results revealed that the ELM with sigmoid activation function (ELM-Sigmoid) was the best among all the other investigated methods (ELM-Hard limit, ELM-Triangular basis, ELM-Sine, ELM-Radial Basis, BPNN and OK). This is because the ELM-sigmoid produced the lowest MAE (0.0175), MSE (0.0005) and RMSE (0.0229) with highest $R^{2}(91.93 \%)$ and $R(95.88 \%)$ respectively. It was concluded that ELM-Sigmoid can be used by field practitioners as a reliable alternative ore grade estimation technique.
\end{abstract}

Keywords: extreme learning machine, artificial intelligence, artificial neural network, grade estimation, kriging

\section{Introduction}

A $\mathrm{n}$ important aspect of mining is ore grade estimation, since it determines the viability of actively mining a mineral of interest. This process mainly involves estimating the reserve and grade using statistical procedures with samples obtained during drilling to determine the feasibility of mining the resource. Geostatistics is the conventional ore grade estimation technique which proves to be effective in the grade prediction of relatively uniform and massive deposits [1-4]. However, in extremely heterogeneous data sets, the geostatistical technique tends to perform poorly due to the complicated nature of the variograms obtained which are mostly rendered useless for further analyses and tend to overestimate or underestimate the resource $[3,5]$. Also, manual tasking during the geostatistical resource estimation processes encourages bias and may introduce errors in the predicted ore grade values. These practical limitations are found in the most widely used geostatistical technique of Ordinary Kriging (OK). Over the years, in the quest to fix and improve the performance of $\mathrm{OK}$, various kriging techniques such as indicator kriging [6,7], disjunctive kriging [8-10], multigaussian kriging [11,12], probability kriging [13-15], lognormal kriging [16,17] and outlier restricted kriging $[18,19]$ were developed. These modifications resulted in more time consuming, computational complexity and overly expensive resource estimation processes. Due to these shortfalls, alternative resource estimation

Received 11 March 2021; revised 16 April 2021; accepted 18 April 2021

Available online 25 May 2021.

* Corresponding author.

E-mail address: caabuntori@umat.edu.gh (C.A. Abuntori). 
Table 1. Review of AI techniques applied in ore grade estimation.

\begin{tabular}{lll}
\hline Author & Technique & Observation \\
\hline Wu and Zhou [20] & $\begin{array}{l}\text { Multilayer Feedforward Neural Network } \\
\text { (MLFNN) with Dynamic Quick-Propagation } \\
\text { (DQP) variant }\end{array}$ & $\begin{array}{l}\text { The technique overestimated and } \\
\text { underestimated low-frequency values such as } \\
\text { high-grade values as a result of smoothing. The } \\
\text { number of data points employed in the study } \\
\text { was 51. }\end{array}$ \\
Al-Alawi and Tawo [22] & BPNN & Required more data for prediction. Hence it
\end{tabular}
Kapageridis and Denby $[23,47]$ Kapageridis et al. [48-50]
Radial Basis Function (RBF) and Multilayer Perceptron (MLP) Kapageridis [24]

Matías et al. [51]

Samanta et al. [52]

Samanta et al. [25]

Chatterjee et al. [35]

Samanta et al. [53]

Mahmoudabadi et al. [26]

Li et al. [21]

Chatterjee et al. [33]

Badel et al. [54]

Guo [55]

Dutta et al. [56]

Dutta et al. [36]

Tahmasebi and Hezarkhani [27] Adaptive Neuro-Fuzzy Inference System (ANFIS)
MLP, Regularisation Networks (RN) and RBF

Kohonen Neural Network (KNN)

MLFNN and SLFN with the Adaboost algorithm, BPNN

\section{ANN}

MLFNN with jump network and Genetic Algorithm (GA)

Levenberg-Marquardt Backpropagation (LMBP) with GA

Wavelet Neural Network (WNN)

GA and k-means clustering NN ensemble with SVM and RBF kernel

MLFNN with Conjugate Gradient Method (CGM) optimisation and K-means clustering

MLP, X-Ray Diffraction (XRD) and Levenberg -Marquardt (LM)

ANN-GA

Support Vector Regression (SVR) and LM Backpropagation (LMBP) NN algorithm
Required more data for prediction. Hence it of sample points used was 163 .

The neural network's resource estimates gave comparable results to kriging with fewer sample data. The number of drill hole data used in these studies ranged from 50 to 3600 .

Kriging outperformed the MLP, RN and RBF using a total of 1932 samples.

KNN and kriging models performed almost equally well. However, grade values were generally overestimated due to the high nugget effect. The total number of drill holes used in the research was 497.

NN generally did not perform well due to the data's low spatial correlation and the high noise of the gold data used. A total of 275 drill hole data was employed in the study.

NN outperformed OK using 5149 data points. OK performed slightly better than the NN model. The number of exploratory borehole data used in the research was 181 .

NNs were quite sensitive when the MLP was used with back propagation-based algorithms (LMBP) in generating initial weight values with a limited training dataset. The study applied a total of 65 drill hole data.

WNN accurately captured the local nonlinearity of the dynamic systems due to its multiscale, multiresolution and localisation ability using 200 drill hole data.

The estimated results obtained using the SVM and RBF outperformed OK. The number of drill hole data applied in the research was 4745 . Results of the Multiple Indicator Kriging (MIK) were more similar to the actual grade values. MIK also had better local precision than the ANN technique using a total number of 1802 data points.

MLP training preferred correlated data. The dataset used was 82 drill holes.

The results obtained from the hybrid NN models generally did not perform well using a total number of 168 borehole data.

The SVR model gave the best results out of the $\mathrm{NN}$, and OK methods applied. However, the upgrade was minimal due to the existence of extreme sample values in the 3500 drill hole data employed in the study.

ANFIS gave better results than FL, ANN and Kriging. The number of data points used in the study was 258 . 
Table 1. (continued)

\begin{tabular}{ll}
\hline Author & Technique \\
\hline Tahmasebi and Hezarkhani [57] & $\begin{array}{l}\text { Multiple layer Neural network based on } \\
\text { Tahmasebi and Hezarkhani [31] }\end{array}$ \\
$\begin{array}{l}\text { (ANN-FA) and ANN-based on Fuzzy lo } \\
\text { Tahmasebi and Hezarkhani [58] }\end{array}$ & $\begin{array}{l}\text { Coactive Neuro-Fuzzy Inference System } \\
\text { (CANFIS) with GA and ANFIS-GA }\end{array}$
\end{tabular}

Maleki et al. [59]

Gholamnejad et al. [32]

Granek [60]

Li et al. [37]

Jafrasteh and Fathianpour [61]

Jafrasteh et al. [34]

Singh et al. [30]

Jahangiri et al. [62]
Support Vector Machine (SVM), Backpropagation Neural Networks (BPNN)

MLFNN with Tanh activation function and Levenberg-Marquardt (LM)

SVM and Convolutional Neural Network (CNN)

Self-adaptive Learning-based Particle Swarm Optimisation Support Vector Regression (SLPSO-SVR) model

Local Linear Radial Basis Function (LLRBF) with Skewed activation function (SG), Simultaneous Perturbation Artificial Bee Colony algorithm (SPABC) and BPNN

Random Forest (RF), Gaussian Process (GP), MLP with LM

Recurrent Neural Network (RNN)

Gustafson-Kessel (GK) clustering algorithm with ANN
Observation

ANN-GA predicted the grade values well with high accuracy than ANN-FL results.

The model had a small and individual structure that allowed the user to manage and control the $\mathrm{NN}$ training to derive the network's sound performance, especially with small data sets. However, due to the user's interference with the processing, the results could be biased. The number of borehole data used was 65 .

The CANFIS-GA produced the best results due to its high correlation coefficient; however, ANFIS-GA gave best the least error on the testing data set. Data from 156 boreholes was applied in the study.

The SVM was fast and gave more accurate results than that of the BPNN model using 4000 data samples.

The predicted values were deemed acceptable and had a correlation coefficient of 0.8 . The number of sample points employed in the study was 2068.

The CNN model was quite complicated, challenging to modify and computationally demanding but had an advantage over SVM by recognising anomalous structures in data. The number of sample points used in the research was 70 .

The SLPSO-SVR technique performed better than PSO-SVR, ANN, comprehensive learning PSO-SVR and Grid-SVR. This technique had many advantages which included its rapid training ability and grade estimation using 2000 sample data points.

The standard RBF trained with SPABC-BP algorithm showed higher generalisation ability and better prediction of ore grades for highly skewed data than LLRBF-SG-SPABC-BP and LLRBF-SPABC-BP. The technique was ideal for capturing nonlinear mappings in the 1250 data points used in the study.

GP gave the best performance compared to the others since it provided a smoother interpolation and offered a more accurate prediction. IK was the next to provide a better estimation, and MLP gave the worse performance. However, all the techniques were sensitive to sudden variations of the copper concentrations. The number of data points employed in the study was 5647.

RNN gave comparable results with kriging. Kriging, however, performed slightly better than RNN. The number of sample points used was 3298 .

The accuracy of the results was poor based on the prediction of some elements; however, predictions were more accurate than the mine's estimation techniques. The number of borehole data applied was 1755 .

(continued on next page) 
Table 1. (continued)

\begin{tabular}{|c|c|c|}
\hline Author & Technique & Observation \\
\hline Manna et al. [63] & $\begin{array}{l}\text { MLP (with } 7 \text { Principal Component Analysis } \\
\text { (PCA) with ADAM optimiser }\end{array}$ & $\begin{array}{l}\text { The obtained results were promising but } \\
\text { required more sample data to ensure the } \\
\text { developed model has a good generalisation } \\
\text { ability as the samples were few ( } 89 \text { samples). }\end{array}$ \\
\hline Zhang et al. [28] & $\begin{array}{l}\text { Weighted Least Square Support Vector } \\
\text { Regression (WLS-SVR) }\end{array}$ & $\begin{array}{l}\text { The robust weighted WLS-SVR outperformed } \\
\text { BPNN, OK and Inverse Distance Weighting } \\
\text { (IDW) due to its strong predictive and } \\
\text { generalisation ability. The number of samples } \\
\text { used the study was } 2304 \text {. }\end{array}$ \\
\hline
\end{tabular}

techniques using Artificial Intelligence (AI) have been applied in ore grade estimation. AI techniques, especially Artificial Neural Networks (ANNs) have effectively been employed in mineral resource estimation using limited data and in highly heterogeneous data sets [20-32]. Most of these techniques applied in literature (Table 1) for ore grade estimation are feedforward neural networks. Their popularity stems from their ability to approximate complex nonlinear mappings between input and output and produce models for a large class of data. From Table 1, the widely used ANN approach in ore grade estimation is the Backpropagation Neural Network (BPNN). Even though majority of the different AI techniques used (Table 1) outperformed kriging [20,22-24,26-28,31,33-37] a few suffered with smoothing in noisy datasets $[20,25,30,34,38]$ which is not ideal in ore grade estimation.

Despite the broad applicability of BPNN, the technique has a wide range of limitations some of which include: overfitting problems, slow convergence and limited reasoning ability. BPNN also requires the model training parameters to be manually tuned in order to obtain optimum results which could lead to local minima with suboptimal solutions [39-41]. The chronological trial and error processes are required in this technique as there are no lied down procedures to ascertain the number of hidden neurons required for the model development $[42,43]$. Thus, the various issues of feedforward neural networks, including slower learning speed due to the use of gradientbased learning algorithms during the training phase and iteratively tuning all network parameters are addressed with the introduction of the Extreme Learning Machine (ELM) in Huang et al. [39].

In light of the strength and mathematical convenience, the study adopted the ELM approach for the ore grade estimation. The ELM was designed for Single Hidden Layer Feedforward Neural Network (SLFN) that randomly chooses its input weights and hidden layer biases and can adequately learn on a given data set. It does this by adopting the function approximation in a finite training set resulting in its ability to apply almost any non-linear activation function to produce distinct predictions [39]. However, in practical application of the ELM, different variants exist based on their activation functions. These activation functions have been found to be a key factor in ELM achieving optimum prediction performance.

Therefore, assessing the impact of the activation function on the ELM performance for ore grade estimation has some scientific value worth investigating. In line with that, this study applied the following activation functions: Radial basis, Hard limit, Sigmoid, Triangular basis and Sine. Despite the wide application of ELM for solving diverse science and engineering problems [44-46], there is close to no application of ELM for ore grade estimation. Furthermore, no ore grade estimation study has assessed and compared the ELM variants with the state-of-the-art benchmark methods of BPNN and OK. Therefore, taking into consideration the strength of the ELM, this paper aims at:

- Determining the viability of the variants of ELM as a novel approach for ore grade estimation using exploratory data from a mine in Ghana;

- Determine its generalisation and predictive ability using heterogeneous data set; and

- Perform comparative analyses between the developed variants of ELM (ELM-Sine, ELMSigmoid, ELM-Radial Basis, ELM-Triangular Basis and ELM-Hard Limit) and benchmark techniques of BPNN and the OK.

\section{Materials and methods}

\subsection{Study area}

This research was conducted in a mine (hereafter Mine X) in Ghana. Mine X deposit is found in the Ashanti belt of the Birimian Supergroup and are 
mainly volcanic rocks. The Birimian supergroup consists of northeast-striking belts with significant faults. The deposits found in the Ashanti belt contain mesothermal gold vein-type deposits [64]. Thus, the mineralisation is found in steep NNESSW to NE-SW trending shears. The gold is found in two principal ore types: quartz veins with freemilling gold, and sulphide ore containing arsenopyrite, pyrite and rare pyrrhotite and marcasite with refractory gold [65]. A map of the study area is shown in Fig. 1.

\subsection{Materials}

Secondary data was obtained from Mine X. The data comprised of assay, survey, lithology, and collar files obtained from exploratory drilling programme. The collar data contains the hole ID, the $X$, $\mathrm{Y}$ and $\mathrm{Z}$ coordinates of each collar, maximum depth of the drill hole and the type of drilling method. The assay data contains the composite Hole ID, From, To and the Assay value observed in that composited section of the borehole. The survey file consists of composite borehole sections at a particular location with its dip and bearing. The lithology file comprises composite collar data and the area between the "From" and "To" is given the observed rock type. The rock types observed were Schist (SC), Meta Volcanic (MV), Quartz (QU), Greywacke (GK), Phyllite (PH) and Laterite (LAT). The entire data set comprised of 3759 drilled holes.

\subsubsection{Statistical description of data}

The samples were initially taken at varying depths which made compositing necessary. Compositing involves the averaging of the original assay values to pre-specified lengths. This creates homogenous support of the data to be used for estimation and minimises data variability. Compositing, therefore, produces more robust statistical and structural analyses. The statistics were done on $1 \mathrm{~m}$ composite samples to identify if populations within the deposit were significantly different. It was also used to access the effects of the data distribution on the methods used for the grade estimations. The total number of samples obtained after compositing was 301507 for the Assay and (X, $Y, Z$ ) coordinate. Table 2 summarises the descriptive statistics of the entire assay data and $(X, Y, Z)$ coordinate. A cumulative frequency graph was also developed to ascertain the data distribution of the assay values (Fig. 2).

\subsection{Methods}

This research applied two primary AI techniques for ore grade estimation, the results of which were then compared with those obtained from OK. The AI techniques used include BPNN and ELM. The ELM for ore grade estimation was assessed based on five different activation functions: Radial basis, Sine, Hard limit, Sigmoid and Triangular basis. The OK technique was carried out using Datamine

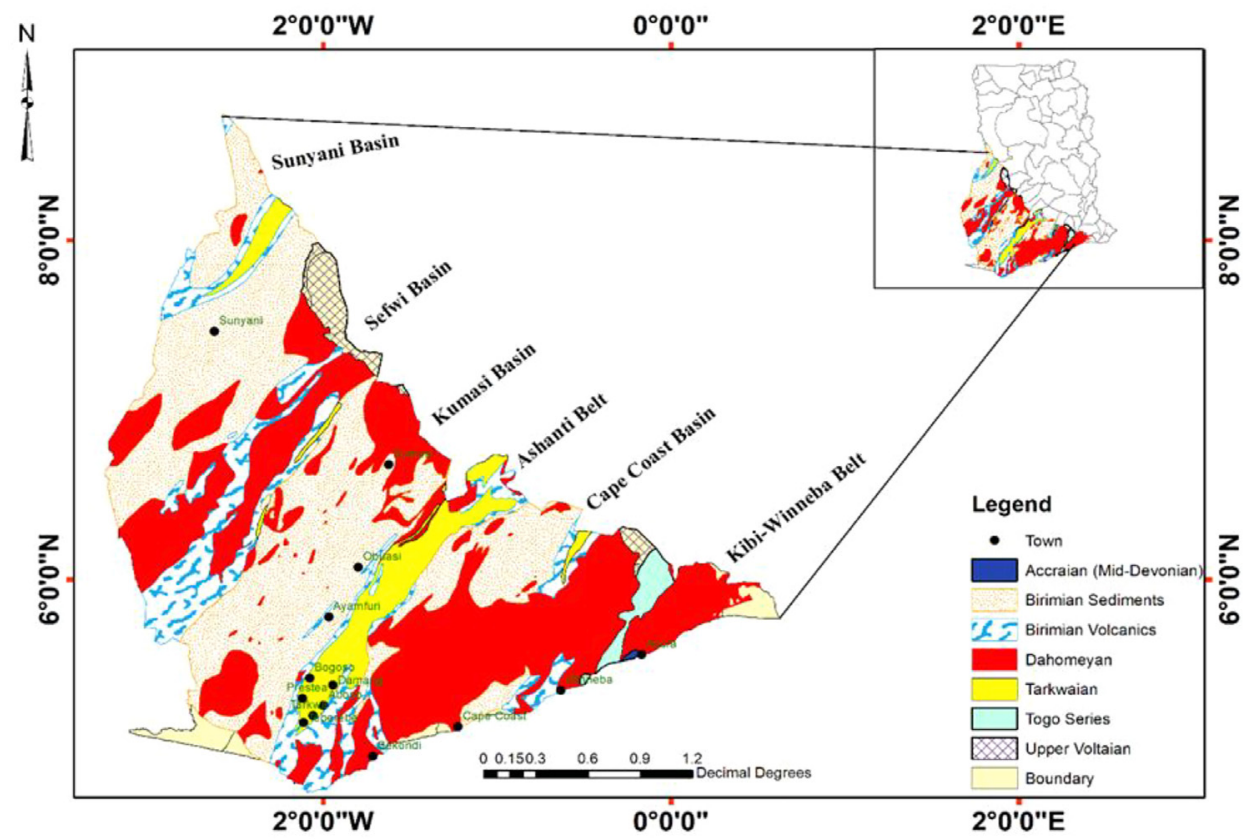

Fig. 1. Geology of the study area. 
Table 2. Descriptive statistics of samples.

\begin{tabular}{lllll}
\hline Field & Assay $(\mathrm{g} / \mathrm{t})$ & X Coordinate $(\mathrm{m})$ & Y Coordinate $(\mathrm{m})$ & $\mathrm{Z}$ Coordinate $(\mathrm{m})$ \\
\hline Minimum & 0 & 11914.702 & 10510.265 & -1604.298 \\
Maximum & 2276 & 13704.413 & 11540.677 & 235.266 \\
Range & 2276 & 1789.711 & 1030.412 & 1839.564 \\
Mean & 4.008 & 12876.910 & 10991.710 & -463.652 \\
Variance & 458.731 & 209383.708 & 10213.987 & 51581.702 \\
Standard Deviation & 21.418 & 457.585 & 101.064 & 227.116 \\
Standard Error & 0.057 & 0.833 & 0.184 & 0.414 \\
Skewness & 49.706 & -0.257 & 0.616 & -0.237 \\
Kurtosis & 3586.004 & -0.871 & 4.209 & 0.810 \\
\hline
\end{tabular}

software, whereas the AI techniques were carried out using MATLAB and Python programs.

\subsubsection{Extreme Learning Machine}

ELM is a learning algorithm for a SLFN which was developed by Huang et al. [39]. ELMs work by iteratively tuning parameters within the network and based on the gaussian probability, it randomly chooses it hidden neurons whiles the Moore-Penrose generalised pseudo inverse is used to analytically determine the output weights of the SLFN [66]. In order to train a SLFN, Eq. (1) [39] is used.

$\min _{\gamma}\left\|R\left(w_{i}, \ldots, w_{\tilde{N}}, b_{i}, \ldots, b_{\tilde{N}}\right) \gamma-Q\right\|$

where $\gamma_{i}=\left[\gamma_{i 1}, \gamma_{i 2}, \ldots, \gamma_{i m}\right]^{Q_{i s}}$ the output weight vector linking the $i$ th hidden node with the output node, $b_{i}$ is the threshold of the $i$ th hidden neuron, $w_{i}=\left[w_{i 1}, w_{i 2}, \ldots, w_{i n}\right]^{Q}$ is the weight vector connecting the $i$ th hidden node and the input neuron. In training a SLFN, the least-squares solution is found using Eq. (2) [67].

$Q=R \gamma$
The smallest norm of output weight is achieved when $\gamma=R^{\dagger} Q$, where $Q$ is the least-squares and $R$ is the output matrix of the hidden layer.

Based on the theory behind ELMs, given $N$ random samples $\left(y_{i}, k_{i}\right)$ where $y_{i}=\left[y_{i 1}, y_{i 2}, \ldots, y_{i n}\right]^{T} \in R^{n}$ and $k_{i}=\left[k_{i 1}, k_{i 2}, \ldots, k_{i m}\right]^{T} \in R^{m},(i=1, \ldots, \tilde{N})$ a standard SLFNs $o_{j}$, with N Nhidden neurons and activation function $g(y)$ applied in the training of the samples are modelled mathematically using Eq. (3) [44].

$\sum_{j=1}^{\tilde{N}} \gamma_{i} g\left(w_{i} \cdot y_{j}+b_{i}\right)=o_{j} \quad j=1,2, \ldots N$

$w_{i} \cdot y_{j}$ represents the inner product of the $w_{i}$ and $y_{j}$ whereby the output weight $w_{i}$ is chosen randomly. Eq. (4) represents the output matrix of the hidden layer [44].

$R=\left[\begin{array}{ccc}g\left(w_{1} \cdot y_{1}+b_{1}\right) & \cdots & g\left(w_{\tilde{N}} \cdot y_{1}+b_{\tilde{N}}\right) \\ \vdots & \cdots & \vdots \\ g\left(w_{1} \cdot y_{N}+b_{1}\right) & \cdots & g\left(w_{\tilde{N}} \cdot y_{N}+b_{\tilde{N}}\right)\end{array}\right]_{N \times \tilde{N}}$

Suppose the number of hidden nodes $\tilde{N}$ is equivalent to the number $N$ of various training data points, $\tilde{N}=N$, the matrix $R$ becomes square and invertible after the input weight vectors $w_{i}$ and the

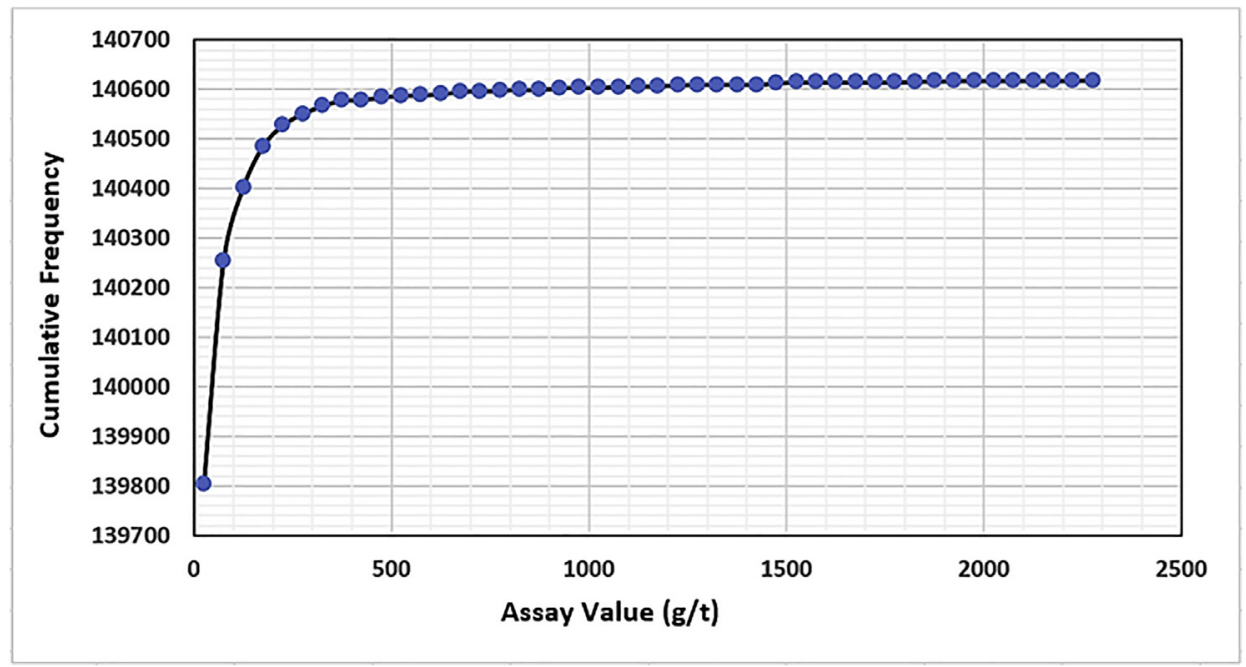

Fig. 2. Cumulative frequency of assay distribution. 
biases $b_{i}$ are selected randomly. Once this happens, SLFNs can estimate the training samples with zero error. None the less $N$ is mostly more than the number of $\tilde{N}$, different training sample data in practical terms. Detailed mathematical background of ELM is produced in [39]. It is important to note that, the ELM prediction performance is based on the type of activation function used.

The main purpose of an activation function is to determine if a neuron should be activated or not, and is achieved by calculating the sum of weights and the addition of a bias. Thus, the non-linearity of the output node is achieved. If an activation function is not applied to a neural network system, the output will act as a simple linear regression function with limited learning ability [68-70]. The selection of the right activation function in a neural network is crucial since an unsuitable activation function can result in the loss of information from the input parameters during forward propagation and consequently, exponential vanishing gradients during backpropagation [71]. Several types of activation functions can be found in literature, however, the most commonly used such as triangular basis, sigmoid, hard limit, sine and radial basis are applied in this study.

The sigmoid activation function also referred to as the logistic function is non-linear and is widely applied in feedforward neural networks [72,73]. The major advantages of the sigmoid activation function is highlighted by Neal [74], some of which include: easiness to understand and its use in shallow networks. The equation for the sigmoid activation function is shown in Eq. (5) [72]:

$g(x)=\frac{1}{1+e^{-x}}$

The sine activation function is sinusoidal in nature. Hence, it varies from the common activation functions as it rises and falls. The study done by Sopena et al. [75] showed that the sinusoids improve accuracy and shortens training time. Although the sine activation function has been applied, this is rarely used as they are difficult to train [76,77]. It is also saturated as its output converges to zero and flattens as $x$ approaches infinity, it also has numerical problems and converges to local minima [78]. The sine activation function is governed by Eq. (6) [78,79]:

$f(x)=\sin (x)$

The Triangular Basis Function (TBF) is a function whose graph is shaped like a triangle, more like an isosceles triangle. It is quite useful in signal processing and when used as an integral transform function produces more realistic signals. The signals from the function fall within the range of -1 to 1 . The triangular basis activation function is expressed in Eq. (7) [80]:

$\widehat{\theta}_{i}(y)=\widehat{r}\left(\frac{y}{y_{i}+1}\right)$

where:

$\widehat{r}(y)= \begin{cases}1-y & \text { if } 0 \leq y \leq 1 \\ 0 & \text { otherwise. }\end{cases}$

$\widehat{\theta}_{i}(y)$ is the TBF and $y$ is an independent variable.

Based on the gaussian curve, the Radial Basis Function (RBF) is achieved. RBF applies a parameter which calculates the mean value of a function. RBF is a real-valued functiongwhose observation is solely dependent on the distance from the origin, thus (Eq. (8)) [72]:

$g(u)=g(\|u\|)$

otherwise, a distance from another fixed-point, i.e. center $c$ (Eq. (9)) [72] results in:

$g(u, c)=g(\|u-c\|)$

Therefore, any function $g$ that satisfies $g(u)=$ $g(\|u\|)$ is a radial function. The Euclidean distance norm and the radial basis function which is commonly taken to be gaussian are merged to obtain an output. The sum of the equations will give Eq. (10) [72] where $y(u)$ is the output and $w_{i}$ is the weight.

$y(u)=\sum_{i=1}^{N} w_{i} g\left(\left\|u-c_{i}\right\|\right)$

The hard limit function is essentially a transfer function that allows the output neuron to produce a 1 if the input attains a threshold, otherwise, it outputs a 0 . It is often used in the perceptron learning rule and as a transfer function, it calculates the output of a layers based on its input. Hard limit activation function is governed by Eq. (11) [81]:

$\operatorname{Hardlimit}(x)=0_{x \leq 0}+1_{x>0}$

\subsubsection{Backpropagation Neural Network}

The AI technique that is commonly used in ore grade estimation is BPNN since it serves as the primary form of neural network $[22,26,28,51,55,59]$. The basic BPNN structure (Fig. 3) has three (3) layers which is made up of the input, hidden and the output; however, multiple hidden layers are accepted in the BPNN architecture. External input 


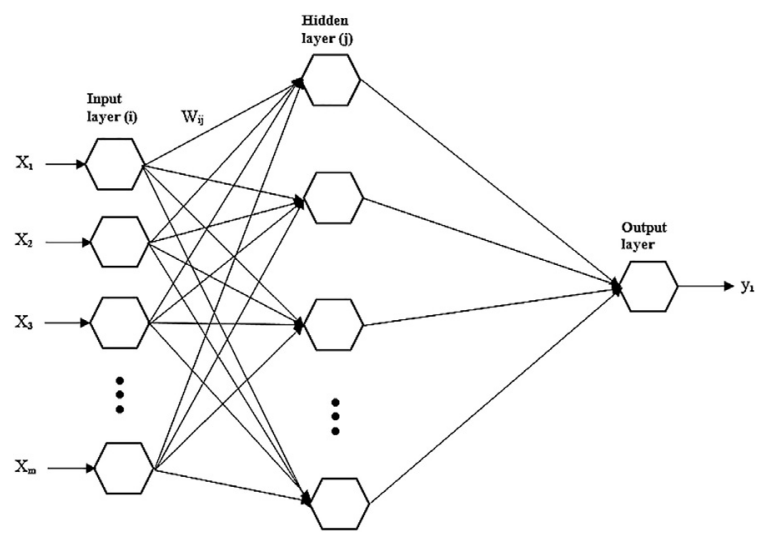

Fig. 3. Basic architecture of BPNN.

parameter are received into the network via the input layer, i.e. $\mathrm{X}, \mathrm{Y}$ and $\mathrm{Z}$ coordinates to each input neuron $X_{j}=\left(X_{1}, X_{2}, X_{3}, \ldots, X_{m}\right)^{T}$ which are assigned specific weights $w_{i j}$ and a bias $b_{i}$ (Eq. (12)) [43]. The input values are then transformed into weighted inputs and are transferred to the hidden layer. A mathematical nonlinear activation function is then used to decide if the data in the input neuron should be activated or not after which the transformed data is given out through the output neuron. As shown in Eq. (12), the input of the output layer is obtained from the output of the hidden layer. The linear activation function is used to transform the input of the hidden layer to the output layer which produces the final network output $y_{i}$.

$y_{i}=f\left(\sum_{j=1}^{m}\left(w_{i j} X_{j}+b_{i}\right)\right)$

Designing a BPNN model involves a critical process of, selecting a suitable number of hidden layers, hidden neurons, training algorithm, and the transfer function. Studies led by several scholars have shown that for solving complicated problems, a BPNN having a single hidden layer is enough as a universal approximator $[43,82,83]$. Hence, one hidden layer was used in this research. The hyperbolic tangent transfer function was employed in the hidden layer whereas the linear transfer function was applied in the output layer to give out the ore grade value for the BPNN model. The training algorithm applied was the Levenberg-Marquardt optimisation method which is primarily used for solving nonlinear least-squares problems. The Levenberg-Marquardt algorithm works by combining the gradient descent and the gaussnewton methods. The gradient descent technique works by updating the parameters in the steepest direction to reduce the sum of squared errors. On the contrary, the Gauss-Newton method is applied, by summing the squared errors and reducing it by assuming the least-squares function to be locally quadratic in parameters, thereby finding the minimum quadratic value [84]. The detailed mathematical background of the Levenberg-Marquardt can be found in [84].

\subsubsection{Ordinary kriging}

Geostatisticians use the variogram as a fundamental tool to measure the spatial continuity of the ore grade data. The experimental variogram is the average variability between samples versus the distance between samples [85]. This variogram model is computed using Eq. (13) [86]:

$\gamma(h)=\frac{1}{2 N(h)} \sum_{\alpha=1}^{N(h)}\left[Z\left(u_{\alpha}+h\right)-Z\left(u_{\alpha}\right)\right]^{2}$

where: $u$ is a vector of coordinates; $z(u)$ is variable under consideration as a function of spatial location; $h$ is the distance between the two points and expressed as a vector; $N(h)$ is the number of pairs found at distance $h$ apart; and $Z(u+h)$ is the value of a second variable at location $h$ units from $u$. The spherical model is widely applied in most orebodies (Fig. 4). This model is characterised by Eqs. (14)-(16) [87].

$\gamma(h)=0$ for $h=0$

$\gamma(h)=C_{0}+C\left[\frac{3}{2}\left(\frac{h}{a}\right)-\frac{1}{2}\left(\frac{h}{a}\right)^{3}\right]$ for $h<a$

$\gamma(h)=C_{0}+C$ for $h \geq a$

where: $a$ is the range and corresponds to the intuitive idea of the range of influence of the regionalised variables. Beyond this value, samples are no longer

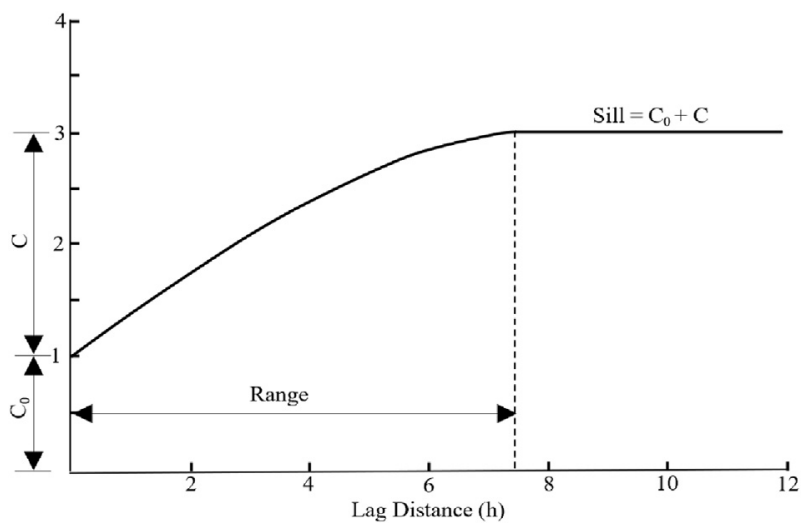

Fig. 4. Spherical semi-variogram model. 
auto-correlated; $C_{0}$ is the nugget variance and represents the random portion of variations of the regionalised variables; $C$ is the spatial variance and is the predictable/structural part of the spatial variance, and $C+C_{0}$ is the Sill.

The grade estimation model can be expressed in the matrix form, as shown in Eq. (17) [86].

$[\mathrm{C}][\mathrm{W}]=[\mathrm{D}]$

where:

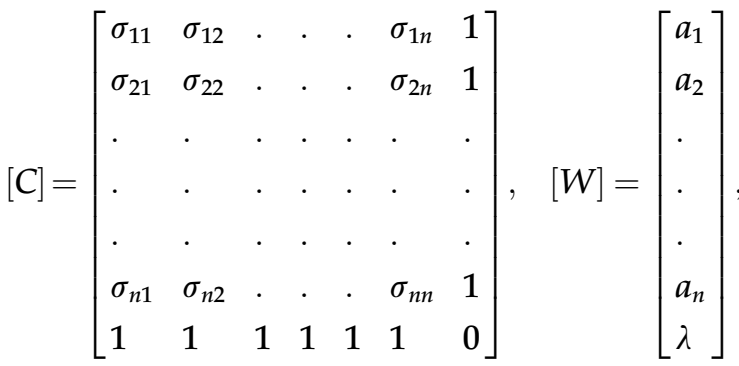

$$
\begin{aligned}
& {[D]=\left[\begin{array}{l}
\sigma V v_{1} \\
\sigma V v_{2} \\
\cdot \\
\cdot \\
\sigma V v_{n} \\
1
\end{array}\right]}
\end{aligned}
$$

In matrix $C, \sigma_{i j}$ is the covariance between any two data points $i$ and $j$; from matrix $D, \sigma V v_{i}$ is the average covariance between a sample and the block to be estimated, while matrix $W$, shows $a_{i}$ which is the weight to be assigned to sample $i$.

\subsection{Statistical evaluation tools}

The efficiency of the predicted results of the various models were compared based on their Correlation Coefficient (R), Mean Square Error (MSE), Mean Absolute Error (MAE), Coefficient of Determination $\left(\mathrm{R}^{2}\right)$ and Root Mean Squared Error (RMSE) which are shown in Eqs (18)-(22) $[28,43,55,88,89]$.

$M S E=\frac{1}{N}\left(\sum_{i=1}^{n}\left(Z_{i}-Z_{i}^{*}\right)^{2}\right)$

$R M S E=\sqrt{\frac{1}{N}\left(\sum_{i=1}^{n}\left(Z_{i}-Z_{i}^{*}\right)^{2}\right)}$
$R^{2}=1-\frac{\sum_{i=1}^{n}\left(Z_{i}-Z_{i}^{*}\right)^{2}}{\sum_{i=1}^{n}\left(Z_{i}-\frac{1}{n} \sum_{i=1}^{n} Z_{i}\right)^{2}}$

$R=\frac{\sum_{i=1}^{n}\left(Z_{i}-\bar{Z}_{i}\right)\left(Z_{i}^{*}-\bar{Z}_{i}^{*}\right)}{\sqrt{\sum_{i=1}^{n}\left(Z_{i}-\bar{Z}_{i}\right)^{2} \times \sqrt{\sum_{i=1}^{n}\left(Z_{i}^{*}-\bar{Z}_{i}^{*}\right)^{2}}}}$

$M A E=\frac{\sum_{i=1}^{n}\left|Z_{i}-Z_{i}^{*}\right|}{N}$

where: $Z$ is the actual assay value obtained from drilling; $Z^{*}$ is the predicted assay value; $\bar{Z}$ is the mean of the actual grade; $\bar{Z}^{*}$ is the mean of predicted grade; $n$ is the sample; and $N$ is the total number of samples.

\subsection{Data preprocessing for model development}

For good predictions using ANN, enough data is required. This study adopted the popular hold-out cross-validation technique commonly employed in ANN modelling to divide the data [43]. As such, the training data set should be larger than the testing data set. Hence, $80 \%$ of the data representing 241206 data points were used for training the network, whereas the remaining $20 \%$ representing 60301 data points were used for testing. The data division was done randomly to prevent bias. However, both divisions possessed similar statistical characteristics to guarantee the models generalisation ability to predict ore grade accurately.

During the data preparation, the data sets were first normalised. This was necessary because, the grade values range between 0 and 2000 while the coordinates range between -1600 to 13700 , which fall in different ranges. Moreover, without the data normalisation the large values (coordinates) essentially influence the results due to its more significant value but may not be more important as a predictor. Therefore, the aim was to change the values of the dataset to a standard scale without distorting the differences in range values and improving the model's validation accuracy. In this research, the scaler applied essentially scaled the data to range from -1 to 1 . The normalisation formula used is expressed in Eq. (23) [90]. 
$p_{i}=p_{\min }+\frac{\left(p_{\max }-p_{\min }\right) \times\left(q_{i}-q_{\min }\right)}{q_{\max }-q_{\min }}$

where: $p_{i}$ is the normalised data, $q_{i}$ is the actual drill hole data, $q_{\max }$ and $q_{\min }$ are the maximum and minimum values of the actual drill hole data with $p_{\min }$ and $p_{\max }$ values set at -1 and 1 , respectively.

\section{Results and discussion}

\subsection{Models developed}

\subsubsection{ELM model for ore grade estimation}

Based on the experimental results, the optimum number of neurons for the developed ELM model was 50. The five activation functions; sigmoid, hard limit, sine, triangular basis and radial basis were applied separately. The optimum ELM model had three input nodes with a single hidden layer made up of 50 hidden neurons and one output with structure [3-50-1]. The three input variables were the $X, Y$, and $Z$ coordinates while the output was the ore grade.

\subsubsection{BPNN model for ore grade estimation}

The input and output data sets used in the ELM model was the same used to develop the BPNN model. Three layers comprising input, hidden and output layers made up the developed BPNN model. As demonstrated in literature, a single hidden layer is capable of approximating any complex problem [82], hence, one hidden layer was employed in this study. The hyperbolic tangent and linear transfer functions were employed in the hidden and output layers to capture both non-linearity and linearity between the input-output data. In training the
BPNN model, the Levenberg-Marquardt algorithm was applied [91]. The best BPNN model obtained in this research has three input nodes, ten neurons in the hidden layer and a single output node, with the structure [3-10-1].

\subsubsection{OK model for ore grade estimation}

As shown in Table 2 the grade distribution shows positive skewness with possible outliers. Therefore, there was the need to apply a top cut to minimise the influence of outliers on the mean and the skewness. The top cut value was based on the log probability curves' (Fig. 5) analysis showing inflexion at specific points, which indicate subpopulation. Hence, the bottom cut value is shown to be at $0.01 \mathrm{~g} / \mathrm{t}$, but due to the high-grade values in the data, the top cut value is at $15 \mathrm{~g} / \mathrm{t}$. Since the deposit is located in the Birimian, and due to the high-grade records in that structure, geologists usually consider the top cut value to be $12 \mathrm{~g} / \mathrm{t}$. However, in this research, $15 \mathrm{~g} / \mathrm{t}$ is considered the top cut value based on the observed inflexion (Fig. 5).

Structural analyses were first performed on the data. The experimental variogram was generated using the exploratory data with a top cut value of $15 \mathrm{~g} / \mathrm{t}$. Based on the experimental points at various lag distances, the variogram parameters were then obtained. The nugget variance $C_{0}=13.4$, spatial variance $C=9.4$ and range $a$ in the $X, Y$ and $Z$ directions were 21.2, 15.03 and 72.54 respectively. These were obtained as the model parameters from the experimental variogram. The spherical model was then superimposed onto the experimental variogram based on the experimental variogram parameters (Fig. 6).

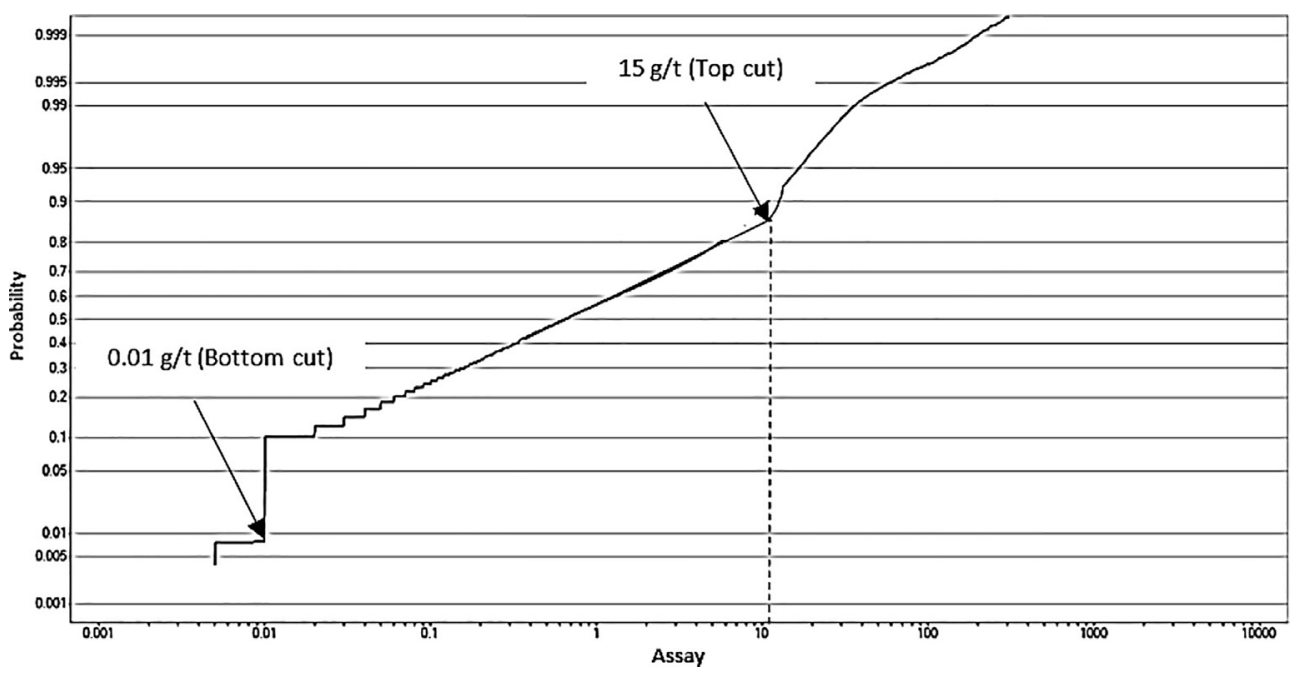

Fig. 5. Log probability plot for assay data. 


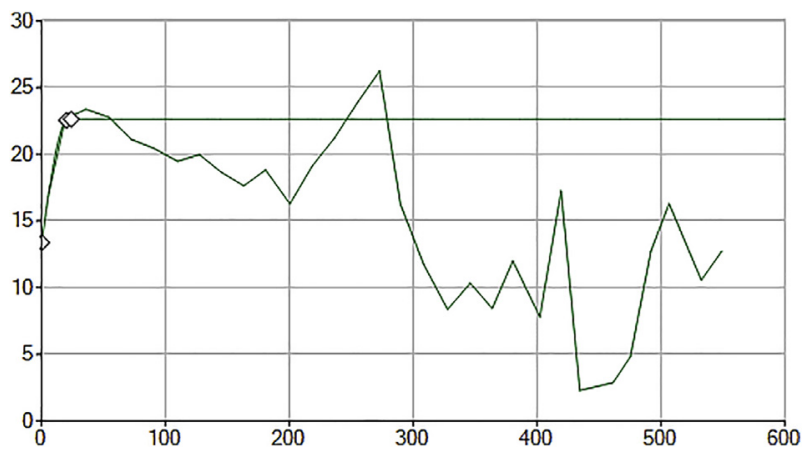

Fig. 6. Directional variogram across strike at $60^{\circ}$.

Based on the results obtained from the structural analyses, ore grade estimation using OK was then conducted. The performance of OK in the ore grade prediction is summarised in Table 4 based on the statistical evaluation tools.

\subsection{Comparative analysis of ELM variants}

In considering the dimensioned error statistic indicators (MSE, MAE and RMSE) as shown in Table 3 , it was observed that the estimation technique, ELM-Sigmoid model obtained the lowest MAE, MSE and RMSE values of 0.0175, 0.000524 and 0.022900 correspondingly. These results (MSE, MAE and RMSE) were interpreted based on the rule of thumb that states that, for a model to approximate closely to the actual data, the error values are closer to zero. The next technique that performed nearly as well as ELM-Sigmoid was ELM-Sine which was closely followed by ELM-Radial basis. The ELMTriangular basis performed fairly while the ELMHard limit gave the poorest results.

The $R^{2}$ (Table 3) indicates that the nearer the value is to 1 or $100 \%$ the better the predicted results. In effect this shows the extent the model applied could explain the prediction variation level from the model compared with actual data. It is observed in Table 3 that the ELM-Sigmoid had the highest $R^{2}$ value of $91.93 \%$, followed by ELM-Sine, $87.91 \%$. The subsequent models: ELM-Radial basis; and ELMTriangular basis performed fairly with $R^{2}$ values measuring $68.86 \%$ and $55.69 \%$ while the ELM-Hard limit performed poorly with $R^{2}$ of $33.37 \%$.

The statistical tool that measures the strength between the relationship of two variables, i.e., actual and estimated values is known as the correlation coefficient $R$, and they fall within the range of -1 to 1. In effect it explains the level of prediction accuracy of the model. In Table 3 , it is noticed that the ELM-Sigmoid and ELM-Sine had $R$ values exceeding 0.9 , making them perform better than the other models, however, ELM-Sigmoid had the highest $R$ of 0.96 . On the contrary, ELM-Radial basis had $R$ of 0.83 while that of ELM-Triangular basis and ELM-Hard limit was 0.75 and below 0.6 respectively. The presented ELM test results are further illustrated in Fig. 7 ((a)-(e)) for visual observation. To this end, it can be stated that the ELM-Sigmoid has demonstrated strong calibration power and best generalisation on the training and testing data with great adaptability as compared to the other models.

Table 3. Optimal train and test results for ELM variants.

\begin{tabular}{|c|c|c|c|c|c|c|c|c|c|c|}
\hline \multirow[t]{3}{*}{ Technique } & \multicolumn{5}{|l|}{ Train } & \multicolumn{5}{|l|}{ Test } \\
\hline & \multicolumn{5}{|c|}{ Performance Criteria } & \multicolumn{5}{|c|}{ Performance Criteria } \\
\hline & $\overline{\mathrm{MAE}}$ & MSE & RMSE & $\mathrm{R}^{2}$ & $\mathrm{R}$ & MAE & MSE & RMSE & $\mathrm{R}^{2}$ & $\mathrm{R}$ \\
\hline ELM-Hard limit & 0.0447 & 0.0043 & 0.0657 & 0.3432 & 0.5858 & 0.0448 & 0.0043 & 0.0657 & 0.3337 & 0.5777 \\
\hline ELM-Triangular basis & 0.0176 & 0.0029 & 0.0539 & 0.5573 & 0.7465 & 0.0360 & 0.0029 & 0.0536 & 0.5569 & 0.7463 \\
\hline ELM-Radial basis & 0.0320 & 0.0021 & 0.0454 & 0.6868 & 0.8287 & 0.0320 & 0.0020 & 0.0449 & 0.6886 & 0.8298 \\
\hline ELM-Sine & 0.0209 & 0.0008 & 0.0285 & 0.8767 & 0.9363 & 0.0212 & 0.0008 & 0.0280 & 0.8791 & 0.9376 \\
\hline ELM-Sigmoid & 0.0172 & 0.0005 & 0.0229 & 0.9201 & 0.9592 & 0.0175 & 0.0005 & 0.0229 & 0.9193 & 0.9588 \\
\hline
\end{tabular}

Table 4. Optimal train and test results.

\begin{tabular}{|c|c|c|c|c|c|c|c|c|c|c|}
\hline \multirow[t]{3}{*}{ Technique } & \multicolumn{5}{|l|}{ Train } & \multicolumn{5}{|l|}{ Test } \\
\hline & \multicolumn{5}{|c|}{ Performance Criteria } & \multicolumn{5}{|c|}{ Performance Criteria } \\
\hline & $\overline{\mathrm{MAE}}$ & MSE & RMSE & $\mathrm{R}^{2}$ & $\mathrm{R}$ & $\overline{M A E}$ & MSE & RMSE & $\mathrm{R}^{2}$ & $\mathrm{R}$ \\
\hline OK & 1.6473 & 7.0565 & 2.6564 & 0.5392 & 0.7343 & 1.6473 & 7.0565 & 2.6564 & 0.5392 & 0.7343 \\
\hline BPNN & 0.0272 & 0.0022 & 0.0464 & 0.5725 & 0.7566 & 0.0555 & 0.0054 & 0.0735 & 0.2866 & 0.5354 \\
\hline ELM-Sigmoid & 0.0172 & 0.0005 & 0.0229 & 0.9201 & 0.9592 & 0.0175 & 0.0005 & 0.0229 & 0.9193 & 0.9588 \\
\hline
\end{tabular}




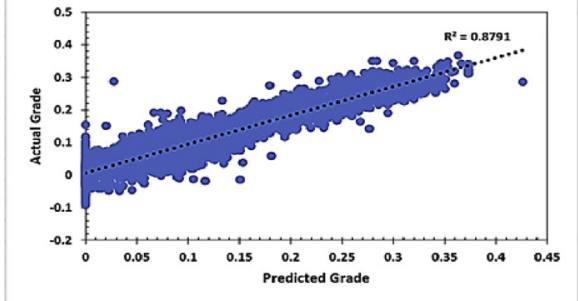

(a)

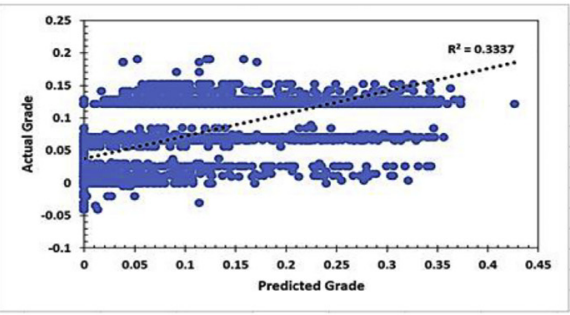

(c)

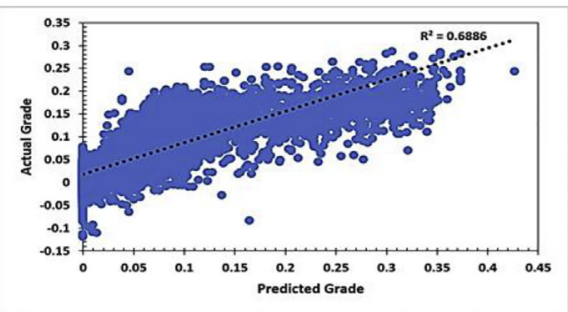

(e)

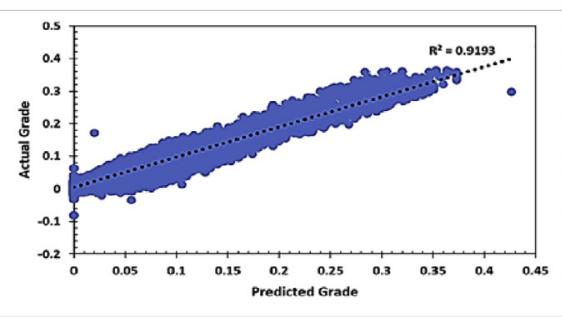

(b)

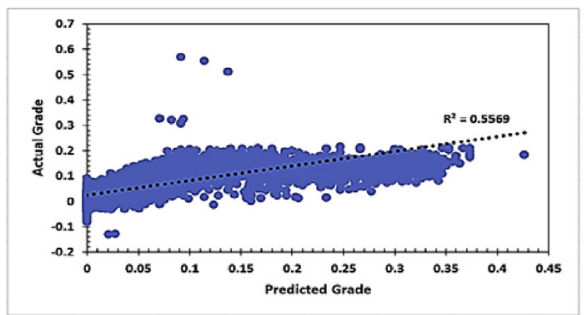

(d)

Fig. 7. Test results: (a) ELM-sine; (b) ELM-Sigmoid; (c) ELM-Hard limit; (d) ELM-Triangular basis; (e) ELM-Radial basis.

\subsection{Performance evaluation of ELM-sigmoid with other investigated techniques}

The ELM-Sigmoid was the best performing model out of the various ELM methods applied in this study. Hence the ELM-Sigmoid is evaluated against state-of-the-art methods of OK and BPNN. The comparison (Table 4) aimed to determine if the proposed ELM-Sigmoid technique could produce comparable or superior results to those obtained

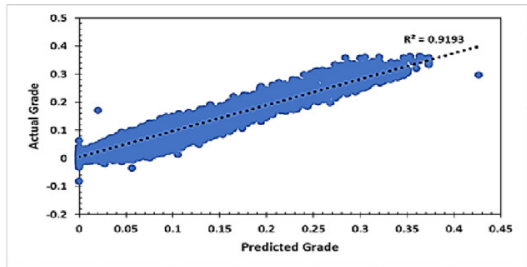

(a)

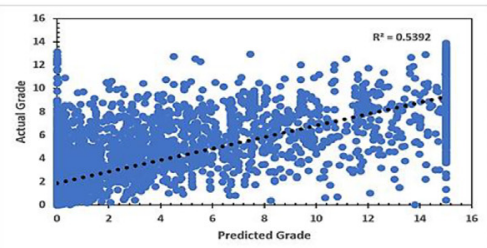

(c)

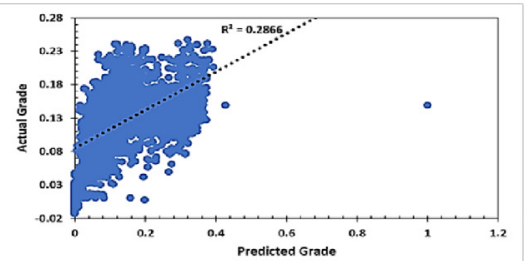

(b)

Fig. 8. Test results: (a) ELM-sigmoid, (b) BPNN and (c) OK. 
from the state-of-the-art established BPNN and OK methods.

Comparatively, ELM-Sigmoid was superior over BPNN and OK as it recorded the lowest errors and had the best performing $R^{2}$ and $R$ values. BPNN had MAE, MSE and RMSE of $0.0555,0.0054$ and 0.0735 whiles OK recorded 1.4127, 6.1599, and 2.4819 respectively. The ELM-Sigmoid had 0.0175, 0.0005, $0.0229,0.9193,0.9588$ for MAE, MSE, RMSE, $R^{2}$ and $R$. A visual comparison of the various models based on the actual and predicted grade values are shown in Fig. 8. The interpretation is that the ELM-Sigmoid model produced a better fitted values to the actual ore grade data than the other methods. The strength of the ELM-Sigmoid comes from the fact that there is less manual tasking (human interference) and minimum fine-tuning adjustable parameters in the model development process. Moreover, optimum predictions were achieved because the ELM technique is not gradient descent based type of algorithm which can trap in local minima and therefore produces global best solutions.

\section{Conclusions}

In this study, ELM has successfully been applied in ore grade prediction of heterogeneous data sets. Five variants of the ELM based on triangular basis, radial basis, hard limit, sine and sigmoid activation functions were developed and tested with data from a mine in Ghana. The proposed ELM techniques were then compared with state-of-the-art established methods of BPNN and the conventional OK. ELM-Sigmoid model generated comparable grade predictions similar to the actual grade values than the other models applied. Thus, the ELM-Sigmoid gave the lowest MAE, MSE and RMSE values of $0.0175,0.0005$ and 0.0229 and highest $R^{2}$ and $R$ of 0.9193 and 0.9588 respectively. Based on the results obtained, it was decided that the proposed ELMSigmoid model has shown encouraging application potential in ore grade estimation and therefore serve as a suitable alternative to the established BPNN and OK models employed in this research. The proposed ELM-Sigmoid model's efficiency was attributed to its inherent ability to randomly select its weights and biases, faster computational speed, less manual tasking in the model development and producing global minimum solutions because it is not a gradient descent type of algorithm where it can trap in local minima.

The developed model for the studied Mine could be adopted as an alternative ore grade estimation tool since OK method which is usually employed produced poor estimates due to the heterogeneous nature of the deposit. Furthermore, the proposed methodology can definitely be replicated for other deposits. This is because it has proven to possess excellent generalisation ability and self-adaptive characteristic feature where it can automatically learn on any given dataset from any Mine.

\section{Ethical statement}

The authors state that the research was conducted according to ethical standards.

\section{Funding body}

This research was funded by Ghana National Petroleum Corporation (GNPC) Professorial Chair in Mining Engineering at the University of Mines and Technology (UMaT), Ghana.

\section{Conflicts of interest}

None declared.

\section{References}

[1] Sinclair AJ, Deraisme J. Geostatistical study of the eagle copper vein, northern British Columbia. Cim Bull 1974;746: 131-42.

[2] Dominy SC, Camm GS, Phelps RFG. Narrow vein mining-A challenge to the operator. Mine Plan Equip Select 1997;97: 125-32.

[3] Roy WD. Using geostatistics to estimate the resources of a narrow vein gold deposit61. Nova Scotia: DalTech; 2000. PhD Dissertation.

[4] Dominy SC, Noppé MA, Annels AE. Errors and uncertainty in mineral resource and ore reserve estimation: the importance of getting it right. Explor Min Geol 2004;11(1-4):77-98. https://doi.org/10.2113/11.1-4.77.

[5] Dominy SC, Annels AE, Camm GS, Wheeler P, Barr SP. Geology in the resource and reserve estimation of narrow vein deposits. Explor Min Geol 1999;6(4):317-33.

[6] Journel AG. Nonparametric estimation of spatial distributions. J Int Assoc Math Geol 1983;15(3):445-68. https:// doi.org/10.1007/BF01031292.

[7] Arslan H. Spatial and temporal mapping of groundwater salinity using ordinary kriging and indicator kriging: the case of Bafra Plain, Turkey. Agric Water Manag 2012;113:57-63. https://doi.org/10.1016/j.agwat.2012.06.015.

[8] Yates SR, Warrick AW, Myers DE. Disjunctive Kriging: 1 Overview of estimation and conditional probability. Water Resour Res 1986;22(5):623-30. https://doi.org/10.1029/ WR022i005p00623.

[9] Daya AA. Application of disjunctive kriging for estimating economic grade distribution in an iron ore deposit: a case study of the Choghart North Anomaly, Iran. J Geol Soc India 2014;83(5):567-76. https://doi.org/10.1007/s12594-014-0085-3.

[10] Mendes MP, Ribeiro L. Nitrate probability mapping in the northern aquifer alluvial system of the river Tagus (Portugal) using Disjunctive Kriging. Sci Total Environ 2010;408(5): 1021-34. https://doi.org/10.1016/j.scitotenv.2009.10.069.

[11] Verly G. The multigaussian approach and its applications to the estimation of local reserves. J Int Assoc Math Geol 1983; 15(2):259-86.

[12] Afzal P, Madani N, Shahbeik S, Yasrebi AB. Multi-Gaussian kriging: a practice to enhance delineation of mineralised 
zones by Concentration-Volume fractal model in Dardevey iron ore deposit, SE Iran. J Geochem Explor 2015;158:10-21. https://doi.org/10.1016/j.gexplo.2015.06.011.

[13] Journel AG. Recoverable reserves estimation-the geostatistical approach. Min Eng 1985;37(6):563-8.

[14] Verly G, Sullivan J. Multigaussian and probability krigingsapplication to the jerritt canyon deposit. Min Eng 1985;37(6): 568-74.

[15] Adhikary PP, Dash CJ, Bej R, Chandrasekharan H. Indicator and probability kriging methods for delineating $\mathrm{Cu}, \mathrm{Fe}$, and Mn contamination in groundwater of Najafgarh Block, Delhi, India. Environ Monit Assess 2011;176(1-4):663-76. https:// doi.org/10.1007/s10661-010-1611-4.

[16] Armstrong M, Boufassa A. Comparing the robustness of ordinary kriging and lognormal kriging: outlier resistance. Math Geol 1988;20(4):447-57. https://doi.org/10.1007/ BF00892988.

[17] Yamamoto JK. On unbiased backtransform of lognormal kriging estimates. Comput Geosci 2007;11(3):219-34. https:// doi.org/10.1007/s10596-007-9046-x.

[18] Arik A. Outlier restricted kriging: a new kriging algorithm for handling of outlier high grade data in ore reserve estimation. In: Proceedings of the 23rd international symposium on the application of compututers and operations research in the.Minerals industry. Littleton: Society of Mining Engineers; 1992. p. 181-7.

[19] Leuangthong O, Nowak M. Dealing with high-grade data in resource estimation. J S Afr Inst Min Metall 2015;115(1): 27-36. https://doi.org/10.17159/2411-9717/2015/v115n1a3.

[20] $\mathrm{Wu} \mathrm{X,} \mathrm{Zhou} \mathrm{Y.} \mathrm{Reserve} \mathrm{estimation} \mathrm{using} \mathrm{neural} \mathrm{network}$ techniques. Comput Geosci 1993;19(4):567-75. https:// doi.org/10.1016/0098-3004(93)90082-G.

[21] Li XL, Xie YL, Guo OJ, Li LH. Adaptive ore grade estimation method for the mineral deposit evaluation. Math Comput Model 2010;52(11-12):1947-56. https://doi.org/10.1016/ j.mcm.2010.04.018.

[22] Al-Alawi SM, Tawo EE. Application of artificial neural networks in mineral resource evaluation. J King Saud Univ Eng Sci 1998;10(1):127-38.

[23] Kapageridis I, Denby B. In: Panagiotou GN, Michalakopoulos TN, editors. Ore grade estimation with modular neural network systems. A case study. Information Technology in the Mineral Industry. vol. 52. Rotterdam: AA Balkema Publ; 1998.

[24] Kapageridis IK. Grade interpolation using radial basis function networks. Mine Plan Equip Select 2002:1-9.

[25] Samanta B, Bandopadhyay S, Ganguli R, Dutta S. A comparative study of the performance of single neural network vs. Adaboost algorithm based combination of multiple neural networks for mineral resource estimation. J S Afr Inst Min Metall 2005;105(4):237-46.

[26] Mahmoudabadi H, Izadi M, Menhaj MB. A hybrid method for grade estimation using genetic algorithm and neural networks. Comput Geosci 2008;13(1):91-101. https://doi.org/ 10.1007/s10596-008-9107-9.

[27] Tahmasebi P, Hezarkhani A. Application of adaptive neurofuzzy inference system for grade estimation; case study, sarcheshmeh porphyry copper deposit, Kerman, Iran. Aust J Basic Appl Sci 2010;4(3):408-20.

[28] Zhang X, Song S, Li J, Wu C. Robust LS-SVM regression for ore grade estimation in a seafloor hydrothermal sulphide deposit. Acta Oceanol Sin 2013;32(8):16-25. https://doi.org/ 10.1007/s13131-013-0337-x.

[29] Badel M, Angorani S, Panahi MS. The application of median indicator kriging and neural network in modeling mixed population in an iron ore deposit. Comput Geosci 2010;37(4): 530-40. https://doi.org/10.1016/i.cageo.2010.07.009.

[30] Singh RK, Ray D, Sarkar BC. Recurrent neural network approach to mineral deposit modelling. In: 4th int. Conference on recent advances on information Technology. 1-5; 2018.

[31] Tahmasebi P, Hezarkhani A. Application of a modular feedforward neural network for grade estimation. Nat
Resour Res 2011;20(1):25-32. https://doi.org/10.1007/s11053011-9135-3.

[32] Gholamnejad J, Kasmaee S, Kohsary A, Nezamolhosseini. A grade estimation of ore stockpiles by using artifi cial neural networks: case study on choghart iron mine in Iran. Int J Min Miner Eng 2012;4(1):17-25. https://doi.org/10.1504/ IJMME.2012.047997.

[33] Chatterjee S, Bandopadhyay S, Machuca D. Ore grade prediction using a genetic algorithm and clustering Based ensemble neural network model. Math Geosci 2010;42(3): 309-26. https://doi.org/10.1007/s11004-010-9264-y.

[34] Jafrasteh B, Fathianpour N, Suárez A. Comparison of machine learning methods for copper ore grade estimation. Comput Geosci 2018;22(5):1371-88. https://doi.org/10.1007/ s10596-018-9758-0.

[35] Chatterjee S, Bhattacherjee A A, Samanta B, Pal SK. Ore grade estimation of a limestone deposit in India using an artificial neural network. Appl GIS 2006;2(1):1-20. https:// doi.org/10.2104/ag060003.

[36] Dutta S, Bandopadhyay S, Ganguli R, Misra D. Machine learning algorithms and their application to ore reserve estimation of sparse and imprecise data. J Intell Learn Syst Appl 2010;2(2):86-96. https://doi.org/10.4236/jilsa.2010.22012.

[37] Li XL, Li LH, Zhang BL, Guo OJ. Hybrid self-adaptive learning based particle swarm optimisation and support vector regression model for grade estimation. Neurocomputing 2013;118:179-90.

[38] Samanta S, Bandopadhyay S, Ganguli R. Data segmentation and genetic algorithms for sparse data division in nome placer gold grade estimation using neural network and geostatistics. Explor Min Geol 2004;11(1-4):69-76.

[39] Huang GB, Zhu QY, Siew CK. Extreme learning machine: a new learning scheme of feedforward neural networks. IEEE Int Joint Conf Neural Network 2004;2:985-90. https:// doi.org/10.1109/IJCNN.2004.1380068.

[40] Huang Y. Advances in artificial neural networks - methodological development and application. Algorithms 2009;2(3) 973-1007. https://doi.org/10.3390/algor2030973.

[41] Adeel A, Larijani H, Javed A, Ahmadinia A. Impact of learning algorithms on random neural network based optimisation for LTE-UL systems. Netw Protoc Algorithm 2015 7(3):157-78. https://doi.org/10.5296/npa.v7i3.8295.

[42] Liu J, Yan K, Zhao X, Hu Y. Prediction of autogenous shrinkage of concretes by support vector machine. Int J Pavement Res Technol 2016;9(3):169-77. https://doi.org/ 10.1016/j.ijprt.2016.06.003.

[43] Arthur CK, Temeng VA, Ziggah YY. Novel approach to predicting blast-induced ground vibration using Gaussian process regression. Eng Comput 2020;36(1):29-42. https:// doi.org/10.1007/s00366-018-0686-3.

[44] Huang GB, Zhu QY, Siew CK. Extreme learning machine: theory and applications. Neurocomputing 2006;70(1-3): 489-501. https://doi.org/10.1016/j.neucom.2005.12.126.

[45] Poerio DV, Brown SD. Localized and adaptive soft sensor based on an extreme learning machine with automated selfcorrection strategies. J Chemometr 2020;34(7):1-18. https:// doi.org/10.1002/cem.3088.

[46] Dash R, Dash PK, Bisoi R. A self adaptive differential harmony search based optimised extreme learning machine for financial time series prediction. Swarm Evol Comput 2014; 19:25-42. https://doi.org/10.1016/j.swevo.2014.07.003.

[47] Kapageridis IK, Denby B. Neural network modellig of ore grade spacial variability. In: International conference on arificial neural networks; 1998. p. 209-14.

[48] Kapageridis IK, Denby B, Hunter G. GEMNET II - a neural ore grade estimation system. In: 29th international symposium on the application of computers and operations research in the minerals industries; 1999. 10pp.

[49] Kapageridis IK, Denby B, Hunter G. Integration of a neural ore grade estimation tool in a 3D resource modeling package. Proc Int Jt Conf Neural Netw 1999;6:3908-12.

[50] Kapageridis IK, Denby B, Schofield D. GEMNET II - an alternative method for grade estimation. 2000. p. 1-8. 
[51] Matías JM, Vaamonde A, Taboada J, González-Manteiga W. Comparison of kriging and neural networks with application to the exploitation of a slate mine. Math Geol 2004;36(4): $463-86$.

[52] Samanta B, Bandopadhyay S, Ganguli R, Dutta S. Sparse data division using data segmentation and kohonen network for neural network and geostatistical ore grade modeling in nome offshore placer deposit. Nat Resour Res 2004;13(3): 189-200.

[53] Samanta B, Ganguli R, Bandopadhyay S. Comparing the predictive performance of neural networks with ordinary kriging in a bauxite deposit Comparing the predictive performance of neural networks with ordinary kriging in a bauxite deposit. Min Technol 2005;114(3):A129-39.

[54] Badel M, Angorani S, Panahi MS. The application of median indicator kriging and neural network in modeling mixed population in an iron ore deposit. Comput Geosci 2011;37(4): 530-40. https://doi.org/10.1016/j.cageo.2010.07.009.

[55] Guo WW. A novel application of neural networks for instant iron-ore grade estimation. Expert Syst Appl 2010;37(12): 8729-35. https://doi.org/10.1016/j.eswa.2010.06.043.

[56] Dutta S, Misra D, Ganguli R, Samanta B, Bandopadhyay S. A hybrid ensemble model of kriging and neural network for ore grade estimation. Int J Surf Min Reclamat Environ 2006; 20(1):33-45. https://doi.org/10.1080/13895260500322236.

[57] Tahmasebi P, Hezarkhani A. Comparison of optimised neural network with fuzzy logic for ore grade estimation. Austr J Basic Appl Sci 2010;4(5):764-72.

[58] Tahmasebi P, Hezarkhani A. A hybrid neural networks-fuzzy logic-genetic algorithm for grade estimation. Comput Geosci 2012;42:18-27. https://doi.org/10.1016/j.cageo.2012.02.004.

[59] Maleki S, Ramazia HR, Moradi S. Estimation of Iron concentration by using a support vector machine and an artificial neural network-the case study of the Choghart deposit southeast of Yazd, Yazd, Iran. Geopersia 2014;4(2):201-12.

[60] Granek J. Application of machine learning algorithms to mineral prospectivity mapping. Vancouver: University of British Columbia; 2016. p. 105. https://doi.org/10.14288/ 1.0340340. PhD Dissertation.

[61] Jafrasteh B, Fathianpour N. A hybrid simultaneous perturbation artificial bee colony and back-propagation algorithm for training a local linear radial basis neural network on ore grade estimation. Neurocomputing 2017;235:217-27. https:// doi.org/10.1016/j.neucom.2017.01.016.

[62] Jahangiri M, Ghavami Riabi SR, Tokhmechi B. Estimation of geochemical elements using a hybrid neural network-Gustafson-Kessel algorithm. J Min Environ 2018;9(2):499-511. https://doi.org/10.22044/jme.2017.5513.1363.

[63] Manna B, Samanta B, Chakravarty D, Dutta D, Chowdhury A, Santra A, et al. Hyperspectral signature analysis using neural network for grade estimation of copper ore. IOP Conf Ser Earth Environ Sci 2018;169(1):1-12.

[64] Smith AJ, Henry G, Frost-Killian S. A review of the birimian supergroup-and tarkwaian group-hosted gold deposits of Ghana. Episodes 2016;39(2):177-97. https://doi.org/10.18814/ epiiugs/2016/v39i2/95775.

[65] Milési JP, Ledru P, Ankrah P, Johan V, Marcoux E, Vinchon C. The metallogenic relationship between Birimian and Tarkwaian gold deposits in Ghana. Miner Deposita 1991; 26(3):228-38. https://doi.org/10.1007/BF00209263.

[66] Cao J, Lin Z, Huang GB. Self-adaptive evolutionary extreme learning machine. Neural Process Lett 2012;36(3):285-305. https://doi.org/10.1007/s11063-012-9236-y.

[67] Liang NY, Huang GB, Saratchandran P, Sundararajan NA. A fast and accurate online sequential learning algorithm for feedforward networks. IEEE Trans Neural Network 2006; 17(6):1411-23. https://doi.org/10.1109/TNN.2006.880583.

[68] Sakthivadivel DA. Formalising the use of the activation function in neural inference. arXiv; 2021. p. 1-7. preprint arXiv: 2102.04896.

[69] Dushkoff M, Ptucha R. Adaptive activation functions for deep networks. Electron Imag 2016;19:1-5. https://doi.org/ 10.2352/ISSN.2470-1173.2016.19.COIMG-149.
[70] Wang Y, Li Y, Song Y, Rong X. The influence of the activation function in a convolution neural network model of facial expression recognition. Appl Sci 2020;10(5):1-20. https:// doi.org/10.3390/app10051897.

[71] Hayou S, Doucet A, Rousseau J. On the impact of the activation function on deep neural networks training. In: International conference on machine learning; 2019. p. 2672-80.

[72] Karlik B, Olgac AV. Performance analysis of various activation functions in generalized MLP architectures of neural networks. Int J Artif Intell Expet Syst 2011;1(4):111-22.

[73] Nwankpa C, Ijomah W, Gachagan A, Marshall S S. Activation Functions: comparison of trends in practice and research for deep learning1-20. arXiv; 2018. Prepr. arXiv1811.03378.

[74] Neal RM. Connectionist learning of belief networks. Artif Intell 1992;56:71-113.

[75] Sopena JM, Romero E, Alquezar R. Neural networks with periodic and monotonic activation functions: a comparative study in classification problems. Inst Elec Eng Conf Pub 1999; 1(470):323-8. https://doi.org/10.1049/cp:19991129.

[76] Wong KW, Leung CS, Chang SJ. Handwritten digit recognition using multi-layer feedforward neural networks with periodic and monotonic activation functions. In: IEEE: In Object recognition supported by user interaction for service robots. 16; 2002. p. 106-9. https://doi.org/10.1109/ icpr.2002.1047806 (3).

[77] McCaughan DB. On the properties of a periodic perceptrons. In: Proceeding of international conference on neural networks. vol. 1; 1997. p. 188-93. https://doi.org/10.1007/ BF01023055.

[78] Lapedes A, Farber R. Nonlinear signal processing using neural networks: prediction and system modelling. Los Alamos National Laboratory: Los Alamos; 1987. p. 1-50.

[79] Gashler MS, Ashmore SC. Modeling time series data with deep Fourier neural networks. Neurocomputing 2016;188: 3-11. https://doi.org/10.1016/j.neucom.2015.01.108.

[80] Melkonian D. Similar basis function algorithm for numerical estimation of Fourier integrals. Numer Algorithm 2010;54(1): 73-100. https://doi.org/10.1007/s11075-009-9324-x.

[81] Huang G, Mao K, Siew CK, Huang DS. Fast modular network implementation for support vector machines. IEEE Trans Neural Network 2005;16(6):1651-63. https://doi.org/ 10.1109/TNN.2005.857952.

[82] Hornik K, Stinchcombe M, White H. Multilayer feedforward networks are universal approximators. Neural Network 1989; 2(5):359-66.

[83] Arthur CK, Temeng VA, Ziggah YY. Soft computing-based technique as a predictive tool to estimate blast-induced ground vibration. J Sustain Min 2019;18(4):287-96. https:// doi.org/10.1016/j.jsm.2019.10.001.

[84] Gavin HP. The levenburg-marqurdt algorithm for nonlinear least squares curve-fitting problems. Durham: Department of Civil and Environmental Engineering, Duke University; 2020. p. 19.

[85] Bhunia GS, Shit PK, Chattopadhyay R. Assessment of spatial variability of soil properties using geostatistical approach of lateritic soil (West Bengal, India). Annal Agra Sci 2018;16(4): 436-43. https://doi.org/10.1016/j.aasci.2018.06.003.

[86] Mert BA, Dag A. A computer program for practical semivariogram modeling and ordinary kriging: a case study of porosity distribution in an oil field. Open Geosci 2017;9(1): 663-74. https://doi.org/10.1515/geo-2017-0050.

[87] Sinclair AJ, Blackwell GH. Applied mineral inventory estimation. Cambridge: Cambridge University Press; 2006. p. 381.

[88] Nezamolhosseini SA, Mojtahedzadeh SH, Gholamnejad J. The application of artificial neural networks to ore reserve estimation at choghart iron ore deposit. Anal Numeric Meth Min Eng 2017;6:73-83.

[89] Asante-Okyere S, Shen C, Ziggah YY, Rulegeya MM, Zhu X. Investigating the predictive performance of Gaussian process regression in evaluating reservoir porosity and 
permeability. Energies 2018;11(12):1-13. https://doi.org/ 10.3390/en11123261.

[90] Mueller AV, Hemond HF. Extended artificial neural networks: incorporation of a priori chemical knowledge enables use of ion selective electrodes for in-situ measurement of ions at environmentally relevant levels. Talanta 2013;117: 112-8. https://doi.org/10.1016/j.talanta.2013.08.045.

[91] Moré JJ. The Levenberg-Marquardt algorithm: implementation and theory. In: Numerical analysis; 1978 p. 105-16. https://doi.org/10.1007/bfb0067700. 